\title{
THE FOUR-SIDED LID DRIVEN SQUARE CAVITY USING STREAM FUNCTION-VORTICITY FORMULATION
}

\author{
Shobha Bagai ${ }^{1}$, Manoj Kumar ${ }^{2}$, Arvind Patel ${ }^{2}$ \\ ${ }^{1}$ Cluster Innovation Centre, University of Delhi, Delhi, India \\ ${ }^{2}$ Department of Mathematics, Faculty of Mathematical Sciences \\ University of Delhi, Delhi, India \\ shobhabagai@gmail.com,manojransiwal@gmail.com,arvindpatelmath09@gmail.com
}

Received: 20 January 2020; Accepted: 13 May 2020

\begin{abstract}
In this paper, an unsteady 2-D incompressible fluid flow with heat and mass transfer in a four-sided lid driven square cavity is investigated numerically. The top, bottom, left, and right walls of the square cavity move to the right, left, downward and upward respectively. All four sides of the cavity move with a uniform velocity. The flow variables are simulated below the critical Reynolds numbers with isothermal and mass-transfer conditions in the square cavity. We have used a streamfunction-vorticity $(\psi-\xi)$ formulation to investigate the fluid flow in terms of flow variables $\psi, \xi, T$ and $C$ at low Reynolds numbers $(R e)$. The Prandtl number $(P r)$ and Schmidt number $(S c)$ have been chosen as 6.62 and 10, 50, 100, 150 respectively, in order to calculate the numerical solutions of $T$ and $C$. The matrix method has been used to evaluate the stability and convergence of the numerical scheme. The conditions obtained from the matrix method have been used to arrive at the numerical solutions with desired accuracy.
\end{abstract}

MSC 2010: 35Q30, 76D05, 76D07,76M12, 76M20

Keywords: four-sided lid-driven square cavity, heat and mass transfer, ADI, isotherms, concentration contours

\section{Introduction}

Numerical simulations of the convective heat and mass transfer problem in a lid driven square cavity have been widely studied due to their broad applications in engineering and physical sciences. Some of the applications involve [1] cooling of electronic devices, float glass production, drying technologies etc. Heat transfer is mainly concerned with the physical process of the transport of thermal energy due to a temperature gradient in an energy equation. Mass transfer is an important topic with vast industrial applications in mechanical, chemical and aerospace engineering. Few of the applications involving mass transfer are absorption and desorption, solvent extraction, evaporation of petrol in internal combustion engines etc.

Ambethkar and Kushawaha [1] have numerically investigated the problem of heat and mass transfer in a rectangular four-sided lid-driven cavity. Ghia et al. [2] have 
used the vorticity-stream function formulation to investigate the lid driven square cavity problem in which the top wall is moving to right while all other walls are kept stationary. Taylor [3] discussed the scraping of viscous fluid from a plane surface. The book of Ottino [4] describes a unified treatment of the mixing of fluids from a kinematical viewpoint, which helps to understand the various mixing problems detected in nature and technology. Luo and Yang [5] have studied the two-sided square cavity problem with an aspect ratio 1.96 with or without heat transfer in terms of streamfunction $(\psi)$ and vorticityfunction $(\xi)$. Ben-Nakhi and Chamkha [6] have investigated the fluid flow around a fine pipe placed in the middle of a square cavity with internal heat generation. Wahba [7], Kumar et al. [8] have numerically investigated the flow behaviour within a two-sided and four-sided lid driven cavity. Wabha has found that the flow field bifurcates from a stable symmetric state to a stable asymmetric state at critical Reynolds numbers 1073 and 129 for a two-sided and four-sided lid driven cavity respectively. Perumal and Dass [8,9] and Azwadi et al. [10] have investigated the steady state solutions of two-dimensional, two- and four-sided lid driven cavity flows in a square cavity using the Lattice Boltzmann Method, the FAS Multigrid method and a time splitting method of the Adams-Bashforth scheme respectively at low Reynolds numbers. Sivasankaran et al. [11] have numerically studied the heat and mass transfer in a top side lid-driven square cavity with sinusoidal heating on its vertical walls. Ambethkar and Kumar [12] have investigated the 2-D unsteady viscous incompressible flow with heat transfer inside a two-sided lid driven square cavity whose top and bottom walls move in opposite directions. Romanò et al. [13] have investigated the incompressible steady solution of a three dimensional flow in a two-sided anti-parallel lid-driven cavity with spanwise-periodic boundary conditions and an aspect ratio $\Gamma=1.7$ using the spectral method on $128^{3}$ grid points. Wu et al. [14] have studied the trajectories of finite-size particles in a two-sided liddriven cavity experimentally. They have found that when the flow is steady and threedimensional, the neutrally buoyant finite-size particles are attracted to periodic orbits. Romano et al. [15-17] have discussed a generic mechanism for finite size coherent particle structures in a two-sided lid-driven cavity. The streamfunction-vorticity formulation, ADI scheme, and Matrix method of analysis are discussed in the books written by Ghoshdastidar [18], Chapra and Canale [19], and Smith [20] respectively.

The main aim of the present work is to investigate the heat and mass transfer inside a four-sided lid driven square cavity using the streamfunction-vorticity formulation. The Alternating-Direction-Implicit (ADI) scheme has been used to discretize the governing equations for the considered problem. To achieve the desired accuracy and prove the stability and convergence of the numerical scheme, we have used the matrix method. 


\section{Mathematical formulation}

The physical model of a four-sided lid driven square cavity with heat and mass transfer of unit size (along with boundary conditions) is illustrated in Figure 1. All four walls of the cavity are maintained at a uniform speed, due to which four vortices of equal size develop symmetrically to both the diagonals namely Top Primary Vortex (TPV), Bottom Primary Vortex (BPV), Left Primary vortex (LPV) and Right Primary Vortex (RPV) as shown in Figure 1. The top and bottom primary vortices move in a clockwise direction, while the left and the right vortices move in an anti-clockwise direction. We have considered the concentration to be zero on the left and right wall of the square cavity, while the top and bottom walls are kept at a constant but different mass flux.

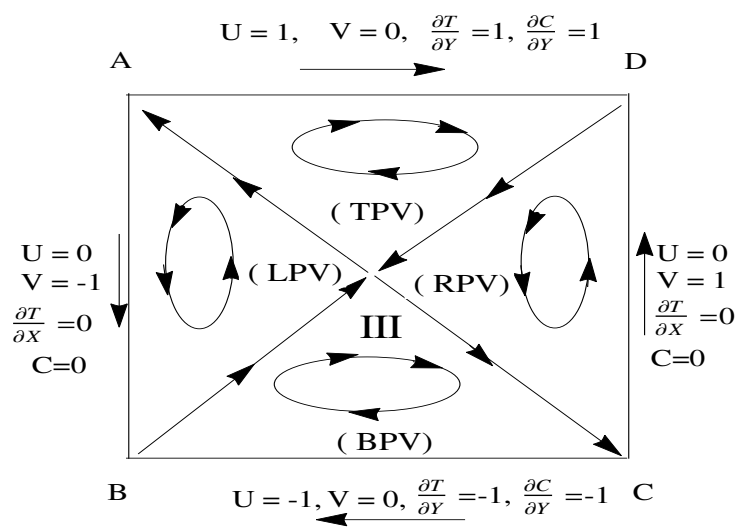

Fig. 1. Four-sided lid driven square cavity

The governing equations of a four-sided lid driven square cavity with heat and mass transfer consist of a continuity equation, $x$ and $y$ momentum equations, energy and mass-transfer equations [12]. The dimensionless governing equations in terms of streamfunction $\psi$ and vorticity $\xi$ using streamfunction-vorticity formulation [18, p. 121] for the considered problem can be expressed as follows:

$$
\begin{gathered}
\frac{\partial^{2} \psi}{\partial x^{2}}+\frac{\partial^{2} \psi}{\partial y^{2}}=-\xi \\
\frac{\partial \xi}{\partial t}+\left(\frac{\partial \psi}{\partial y}\right)\left(\frac{\partial \xi}{\partial x}\right)-\left(\frac{\partial \psi}{\partial x}\right)\left(\frac{\partial \xi}{\partial y}\right)=\frac{1}{R e}\left(\frac{\partial^{2} \xi}{\partial x^{2}}+\frac{\partial^{2} \xi}{\partial y^{2}}\right), \\
\frac{\partial T}{\partial t}+\left(\frac{\partial \psi}{\partial y}\right)\left(\frac{\partial T}{\partial x}\right)-\left(\frac{\partial \psi}{\partial x}\right)\left(\frac{\partial T}{\partial y}\right)=\frac{1}{P r}\left(\frac{\partial^{2} T}{\partial x^{2}}+\frac{\partial^{2} T}{\partial y^{2}}\right),
\end{gathered}
$$




$$
\frac{\partial C}{\partial t}+\left(\frac{\partial \psi}{\partial y}\right)\left(\frac{\partial C}{\partial x}\right)-\left(\frac{\partial \psi}{\partial x}\right)\left(\frac{\partial C}{\partial y}\right)=\frac{1}{S c}\left(\frac{\partial^{2} C}{\partial x^{2}}+\frac{\partial^{2} C}{\partial y^{2}}\right) .
$$

where $\psi, \xi, T$ and $C$ are the stream function, vorticity, temperature and concentration respectively. The non-dimensional parameters are Reynolds number $R e=U L / v, L$ is the length of the square cavity, $v$ is kinematic viscosity; Prandtl number $\operatorname{Pr}=v / \alpha$, $\alpha$ is thermal diffusivity; and the Schmidt number $S c=v / D, D$ is mass diffusivity. The boundary conditions are given by: lower plate moves leftwards $\psi=0, \partial \psi / \partial y=$ $=-1, \partial \psi / \partial x=0, \partial T / \partial y=-1, \partial C / \partial y=-1$; the upper plate moves rightwards $\psi=0, \partial \psi / \partial y=1, \partial \psi / \partial x=0, \partial T / \partial y=1, \partial C / \partial y=1$; the right plate moves upwards, $\psi=0, \partial \psi / \partial y=0, \partial \psi / \partial x=-1, \partial T / \partial x=0, C=0$; the left plate moves downwards $\psi=0, \partial \psi / \partial y=0, \partial \psi / \partial x=1, \partial T / \partial x=0, C=0$.

\section{Numerical discretization}

The governing equations of the considered problem are discretized by using the finite difference method, namely the Alternating-Direction-Implicit (ADI) method. Using the finite difference formula [19, p. 883] and ADI Scheme, the discretized eqs. (1)-(3) at different time steps are given by Ambethkar and Kumar [12, p. 462-463]. Similarly, the mass transfer equation (4) can be discretized as

$$
\begin{aligned}
& {\left[\frac{\Delta t}{8 \Delta x \Delta y}\left(\psi_{i+1, j}^{t}-\psi_{i-1, j}^{t}\right)-\frac{\Delta t}{2 S c \Delta y^{2}}\right] C_{i, j-1}^{t+\frac{1}{2}}+\left[1+\frac{\Delta t}{S c \Delta y^{2}}\right] C_{i, j}^{t+\frac{1}{2}}+} \\
& {\left[-\frac{\Delta t}{8 \Delta x \Delta y}\left(\psi_{i+1, j}^{t}-\psi_{i-1, j}^{t}\right)-\frac{\Delta t}{2 S c \Delta y^{2}}\right] C_{i, j+1}^{t+\frac{1}{2}}=\left[\frac{\Delta t}{8 \Delta x \Delta y}\left(\psi_{i, j+1}^{t}-\psi_{i, j-1}^{t}\right)+\frac{\Delta t}{2 S c \Delta x^{2}}\right]} \\
& \times C_{i-1, j}^{t}\left[+1-\frac{\Delta t}{S c \Delta x^{2}}\right] C_{i, j}^{t}+\left[-\frac{\Delta t}{8 \Delta x \Delta y}\left(\psi_{i, j+1}^{t}-\psi_{i, j-1}^{t}\right)+\frac{\Delta t}{2 S c \Delta x^{2}}\right] C_{i+1, j}^{t} \cdot \\
& {\left[-\frac{\Delta t}{2 S c \Delta x^{2}}-\frac{\Delta t}{8 \Delta x \Delta y}\left(\psi_{i, j+1}^{t}-\psi_{i, j-1}^{t}\right)\right] C_{i-1, j}^{t+1}+\left[1+\frac{\Delta t}{S c \Delta x^{2}}\right] C_{i, j}^{t+1}+} \\
& {\left[-\frac{\Delta t}{2 S c \Delta x^{2}}+\frac{\Delta t}{8 \Delta x \Delta y}\left(\psi_{i, j+1}^{t}-\psi_{i, j-1}^{t}\right)\right] C_{i+1, j}^{t+1}=\left[\frac{\Delta t}{2 S c \Delta y^{2}}-\frac{\Delta t}{8 \Delta x \Delta y}\left(\psi_{i+1, j}^{t}-\psi_{i-1, j}^{t}\right)\right]} \\
& \times C_{i, j-1}^{t+\frac{1}{2}}+\left[1-\frac{\Delta t}{S c \Delta y^{2}}\right] C_{i, j}^{t+\frac{1}{2}}+\left[\frac{\Delta t}{2 S c \Delta y^{2}}+\frac{\Delta t}{8 \Delta x \Delta y}\left(\psi_{i+1, j}^{t}-\psi_{i-1, j}^{t}\right)\right] C_{i, j+1}^{t+\frac{1}{2}} \cdot
\end{aligned}
$$

These discretized equations are solved iteratively through an algorithm by stream function-vorticity formulation [12, p. 467]. We solve the mass transport equation (4) for $C$ at all interior grid points after Step (5), and then calculate the local and average Sherwood number after obtaining the average Nusselt-number. 


\section{Stability and convergence of the numerical scheme}

The matrix method is used to prove the stability and convergence of the considered problem. Using the finite difference approximation formula described in [12, p. 464] and assuming $A^{*}=D_{0, x}^{2}, B^{*}=D_{0, y}^{2}, C^{*}=D_{0, x}, D^{*}=D_{0, y}$; the discretized governing equations can be expressed in matrix form as follows

$$
\left[\begin{array}{l}
\xi_{i, j}^{t+1} \\
T_{i, j}^{t+1} \\
C_{i, j}^{t+1}
\end{array}\right]=\left[\begin{array}{ccc}
F_{1} E_{1} & 0 & 0 \\
0 & F_{2} E_{2} & 0 \\
0 & 0 & F_{3} E_{3}
\end{array}\right]\left[\begin{array}{c}
\xi_{i, j}^{t} \\
T_{i, j}^{t} \\
C_{i, j}^{t}
\end{array}\right]
$$

where

$$
\begin{aligned}
& E_{1}=\left[I+\frac{k}{2} v_{i, j} D^{*}-\frac{k}{2 R e} B^{*}\right]^{-1}\left[I-\frac{k}{2} u_{i, j} C^{*}+\frac{k}{2 R e} A^{*}\right], \\
& F_{1}=\left[I+\frac{k}{2} u_{i, j} C^{*}-\frac{k}{2 R e} A^{*}\right]^{-1}\left[I-\frac{k}{2} v_{i, j} D^{*}+\frac{k}{2 R e} B^{*}\right] . \\
& E_{2}=\left[I+\frac{k}{2} v_{i, j} D^{*}-\frac{k}{2 P r} B^{*}\right]^{-1}\left[I-\frac{k}{2} u_{i, j} C^{*}+\frac{k}{2 P r} A^{*}\right], \\
& F_{2}=\left[I+\frac{k}{2} u_{i, j} C^{*}-\frac{k}{2 P r} A^{*}\right]^{-1}\left[I-\frac{k}{2} v_{i, j} D^{*}+\frac{k}{2 P r} B^{*}\right] . \\
& E_{3}=\left[I+\frac{k}{2} v_{i, j} D^{*}-\frac{k}{2 S c} B^{*}\right]^{-1}\left[I-\frac{k}{2} u_{i, j} C^{*}+\frac{k}{2 S c} A^{*}\right], \\
& F_{3}=\left[I+\frac{k}{2} u_{i, j} C^{*}-\frac{k}{2 S c} A^{*}\right]^{-1}\left[I-\frac{k}{2} v_{i, j} D^{*}+\frac{k}{2 S c} B^{*}\right] .
\end{aligned}
$$

To prove the stability of the considered scheme by the matrix method [20], norm of the above matrix must be less than or equal to 1 . This requires $\left\|F_{1} E_{1}\right\| \leq 1,\left\|F_{2} E_{2}\right\| \leq$ 1 and $\left\|F_{3} E_{3}\right\| \leq 1$. Now, proceding on the similar lines as desribed by Ambethkar and Kumar [12] to prove the unconditional stability of the numerical scheme, we have $\left|u_{i, j} \Delta x \operatorname{Re}\right| \leq 2,\left|v_{i, j} \Delta y \operatorname{Re}\right| \leq 2, \Delta t<\operatorname{Re} \Delta x^{2} ; \quad\left|u_{i, j} \Delta x \operatorname{Pr}\right| \leq 2,\left|v_{i, j} \Delta y \operatorname{Pr}\right| \leq 2, \Delta t<$ $\operatorname{Pr} \Delta x^{2}$; and $\left|u_{i, j} \Delta x S c\right| \leq 2,\left|v_{i, j} \Delta y S c\right| \leq 2, \Delta t<S c \Delta x^{2}$.

\section{Results and discussion}

The numerical solutions of the unknown flow variables are calculated by using finite difference method namely Alternating-Direction-Implicit (ADI) scheme at low Reynolds numbers with suitable Prandtl number $(\mathrm{Pr})$ and Schmidt number $(\mathrm{Sc})$ for the considered problem. The Prandtl number $P r=6.62, T_{0}=10$ and $C_{0}=0$ is fixed throughout the calculation. This section presents the streamlines, isotherms, vorticity, 
concentration contours, locations of the streamline vortices inside the cavity, and the horizontal, vertical velocities along the vertical, horizontal line through the geometric center of the square cavity.

The streamline contours of the four-sided, lid-driven cavity have been depicted in Figure 2 for different Reynolds numbers. At the end of numerical simulation, the four vortices are generated as explained in Section 2. Figure 2 shows the streamfunction values on these primary vortices are the same in magnitude but may have negative or positive values. A positive value shows that the vortex is moving in an anti-clockwise direction, while a negative value indicates the clockwise direction.
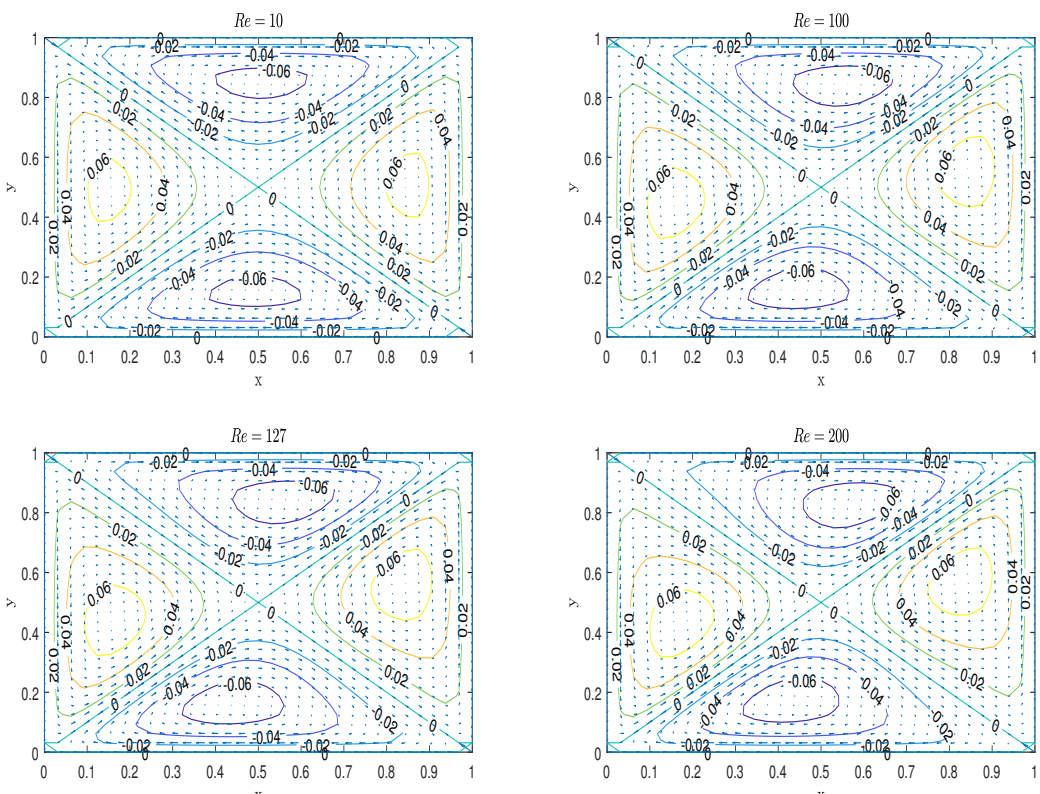

Fig. 2. Streamline contours for four-sided lid-driven square cavity at different Reynolds numbers: $R e=10, R e=100, R e=127$, and $R e=200$

The center of streamline vortices starts shifting towards anti-diagonal (the line joining left-bottom corner and top-right corner of the square cavity) as the Reynolds number increases from $R e=10$ to 127 . Table 1 presents the location of the centers of the streamline vortices for the considered problem at different Reynolds numbers. The center of TPV starts shifting to the right and slightly downwards which is at $(0.613,0.840)$, and the RPV starts shifting upward and slightly to the left at $(0.840,0.613)$ as the Reynolds number increases from $R e=10$ to 200 . The center of BPV starts shifting towards left and slightly upwards which is at $(0.387,0.167)$, and the LPV starts shifting towards right and slightly downwards at $(0.167,0.387)$ as the Reynolds number increases $R e=10$ to 200. Notice that the center of streamline vortices of BPV and LPV moves in opposite directions to TPV and RPV respectively with an increase of the Reynolds number. 
Table 1. Locations of the centers of the streamline vortices for four-sided lid-driven square cavity at different Reynolds numbers

\begin{tabular}{|c|c|c|c|c|}
\hline $\begin{array}{c}\text { Reynolds } \\
\text { number }\end{array}$ & $\begin{array}{c}\text { Top Primary } \\
\text { Vortex (TPV) }\end{array}$ & $\begin{array}{c}\text { Bottom Primary } \\
\text { Vortex (BPV) }\end{array}$ & $\begin{array}{c}\text { Left Primary } \\
\text { Vortex (LPV) }\end{array}$ & $\begin{array}{c}\text { Right Primary } \\
\text { Vortex (RPV) }\end{array}$ \\
\hline 10 & $0.509,0.849$ & $0.497,0.157$ & $0.157,0.497$ & $0.849,0.509$ \\
\hline 100 & $0.560,0.842$ & $0.446,0.160$ & $0.160,0.446$ & $0.842,0.560$ \\
\hline 127 & $0.567,0.840$ & $0.440,0.167$ & $0.167,0.440$ & $0.840,0.567$ \\
\hline 200 & $0.613,0.840$ & $0.387,0.167$ & $0.167,0.387$ & $0.840,0.613$ \\
\hline
\end{tabular}

Figures 3 and 4 recognize that the absolute value of velocity is maximum near the walls of a square cavity due to the movement of the walls of the square cavity. The direction and magnitude of the velocity profiles changes as we move towards the center of the cavity due to fluid circulation. The absolute value of the velocity profiles increases in the vicinity of the moving wall of the square cavity as the Reynolds number increases from $R e=10$ to 200 .

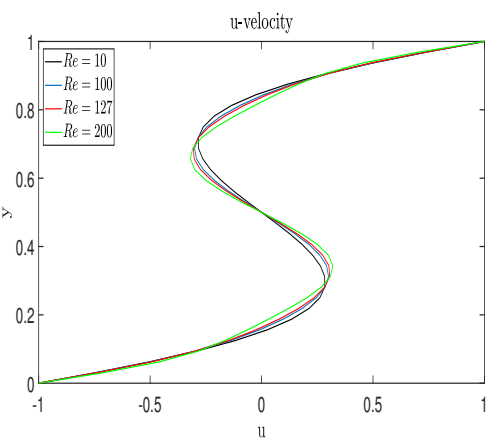

Fig. 3. u-velocity profiles along $x=0.5$

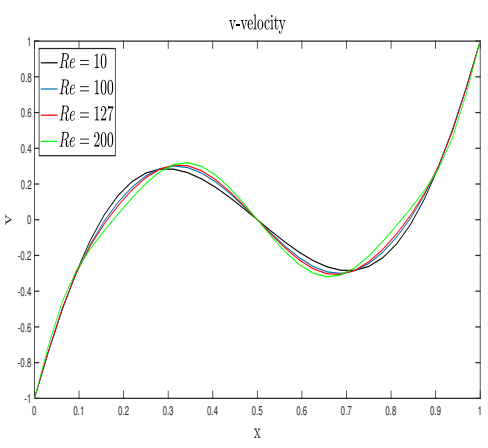

Fig. 4. v-velocity profiles along $y=0.5$

Figure 5 shows that the vorticity contours in the vicinity of the top and bottom walls are negative in sign, while in the vicinity of the left and right walls, the vorticity contours are positive in sign. A negative sign represents the clockwise rotation while a positive sign represents an anti-clockwise rotation. The vorticity contours are symmetric about the horizontal as well as the vertical line through the geometric center of the square cavity for Reynolds number $R e=10$, while these are the mirror images for $R e=100$ to 200 . The absolute value of vorticity along the diagonal as well as anti-diagonal of the square cavity increases with an increase of Reynolds number $R e=10$ to 200 . The vorticity contours start shifting towards anti-diagonal as well as the geometric center of the square cavity with an increase of the Reynolds number.

Figure 6 illustrates the isotherms contours are symmetric about the horizontal line through the geometric center of the square cavity and are wavy. The isotherms contours increase from the geometric center of the square cavity towards the top and bottom wall at a particular Reynolds number. The temperature profiles increase with an increase of the Reynolds number from $R e=10$ to 100 , while it decreases for $R e=100$ to 200 . The temperature profiles are symmetric about the horizontal line through the geometric center of the cavity. 

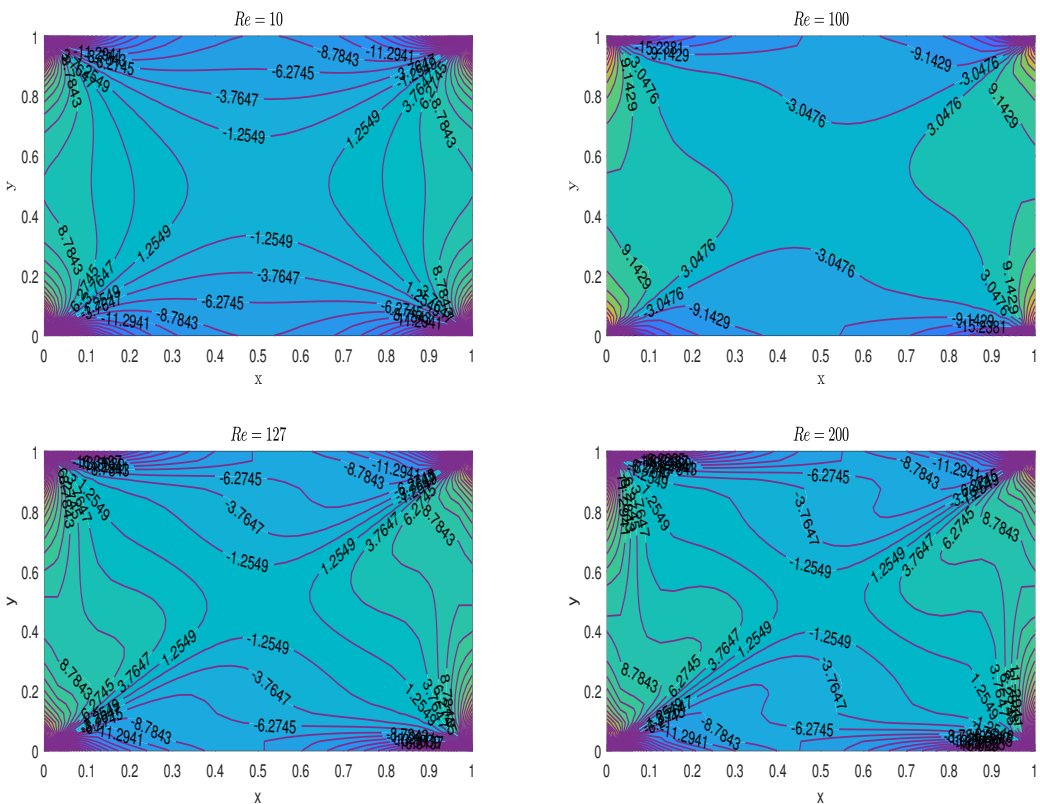

Fig. 5. Vorticity contours for four-sided lid-driven square cavity at different Reynolds numbers: $R e=10, R e=100, R e=127$, and $R e=200$
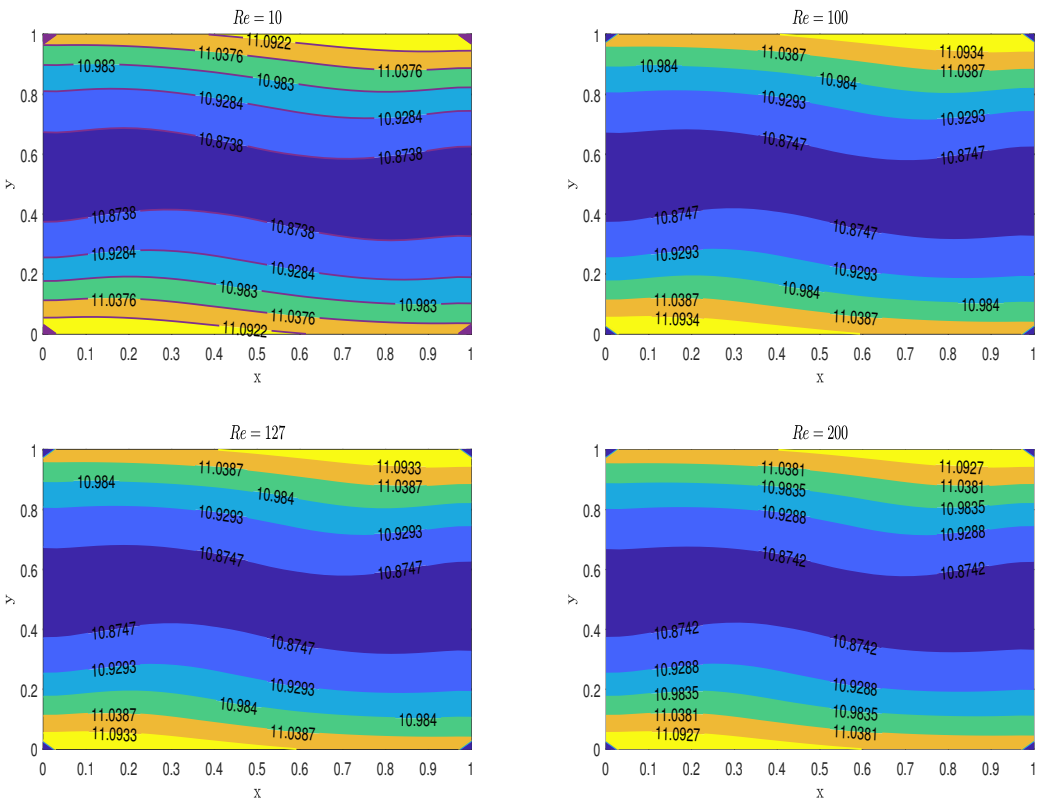

Fig. 6. Isotherms contours for four-sided lid-driven square cavity at different Reynolds numbers: $R e=10, R e=100, R e=127$, and $R e=200$ 
Figure 7 illustrates the temperature and average Nusselt number along the vertical line through the geometric center of the square cavity for different Reynolds numbers and at a distinct time level. The temperature of the fluid is maximum in the vicinity of the top and bottom wall, and it decreases towards the geometric center of the square cavity at a particular Reynolds number. The temperature profiles are symmetric about the horizontal line through the geometric center of the cavity. The temperature starts increasing with an increase of Reynolds numbers $R e=10$ to 127 , while it starts decreasing from $R e=127$ to 200 at time $t=3$. Temperature increases with an increase of time from $t=1$ to 3 . The average Nusselt number along the vertical line through the geometric center of the square cavity decreases and attains the steady-state solution at Reynolds number $R e=10$, while it decreases and slightly increases to achieve the steady-state solution for $R e=100$ to 200 . The maximum absolute value of the average Nusselt number increases with an increase of Reynolds numbers $R e=10$ to 200 .
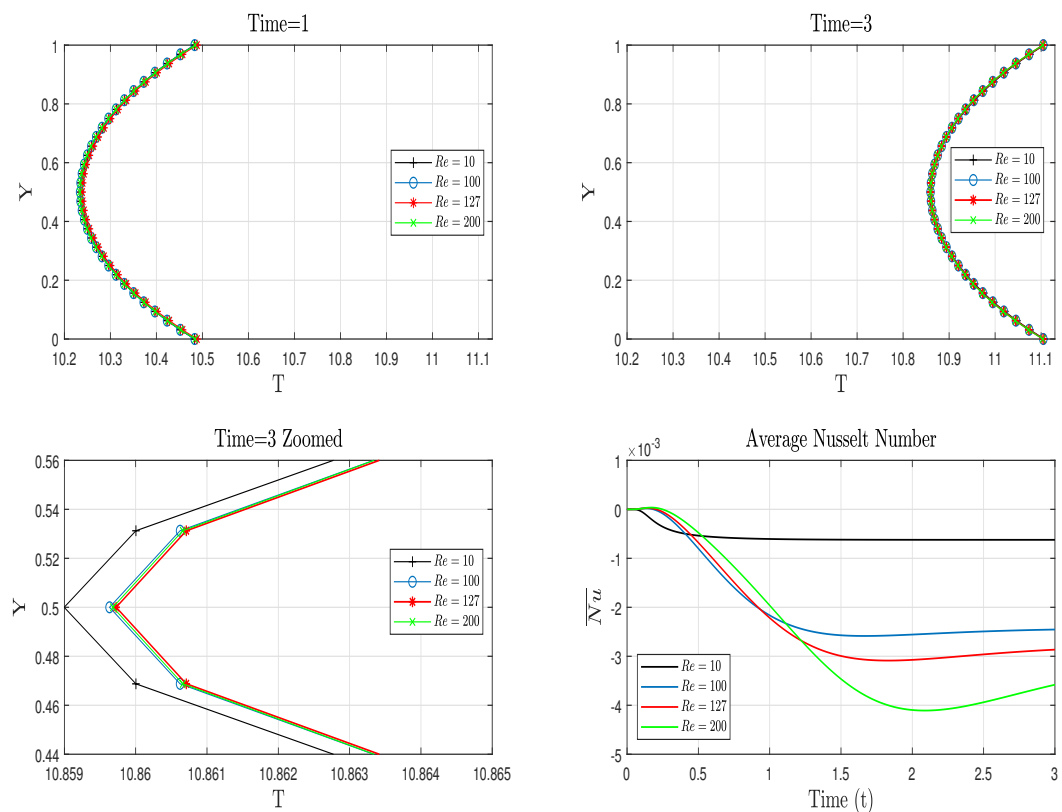

Fig. 7. Temperature and average Nusselt number along vertical line through the geometric center of the square cavity at different Reynolds numbers $R e=10,100,127,200$ and different time level $t=1,3$

The concentration contours of the four-sided lid-driven square cavity have been depicted in Figure 8 with Reynolds number $R e=100$ and different Schmidt numbers (a) $S c=10$, (b) $S c=50$ (c) $S c=100$, (d) $S c=150$. Since the concentration is zero on the left and right wall of the square cavity, the only contribution to concentration is due to the mass flux at the top and bottom wall. The concentration from the left and right wall of the cavity increases towards the geometric center of the square cavity at a particular Schmidt number. The concentration contours look like symmetric about the horizontal line through the geometric center of the square cavity at $S c=10$, 

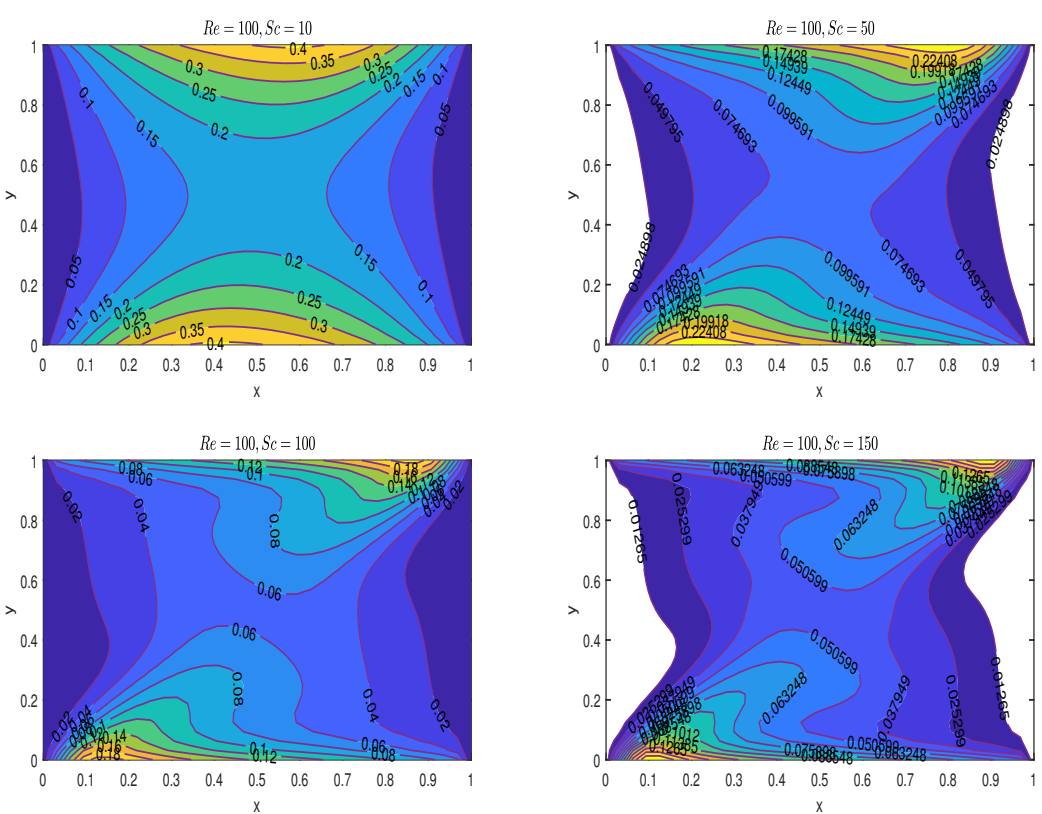

Fig. 8. Concentration contours for four-sided lid-driven square cavity with $R e=100$ and different Schmidt numbers: $S c=10, S c=50, S c=100$, and $S c=150$

while the contours are the mirror image of each other about the horizontal and vertical line through the geometric center of the square cavity for Schmidt numbers $S c=50$ to 150 . The concentration contours start shifting anti-diagonally and towards the geometric center of the square cavity as the Schmidt number increases from $R e=10$ to 150 . The absolute value of concentration decreases with an increase of Schmidt number $S c=10$ to 150 .

Figure 9 illustrates the concentration along the vertical line through the geometric center of the square cavity for different Reynolds numbers, Schmidt number, and at distinct time levels. The effect of the Reynolds number shown in the first three subfigures by ranging the Reynolds number $R e=10$ to 200 and Schmidt number 10. The last subfigure illustrates the effect of Schmidt number $S c=10$ to 150 and Reynolds number $R e=100$. The concentration profiles behave alike as the temperature profile for Reynolds numbers $R e=10$ to 200 and Schmidt number $S c=10$ at time $t=1$ and 3 .

The concentration profile is smooth for $S c=10$, while it starts oscillating with an increase of Schmidt number $S c=10$ to 150 . The absolute value of concentration along the vertical line through mid of the square cavity reduces with an increase of Schmidt number $S c=10$ to 150 and Reynolds number $R e=100$.

The effect of Reynolds numbers and Schmidt numbers on the average Sherwood number along the vertical line through the geometric center of the square cavity has been described in Figure 10. The average Sherwood number decreases with an increase of Reynolds number $R e=10$ to 200 and $S c=10$. At Reynolds number 

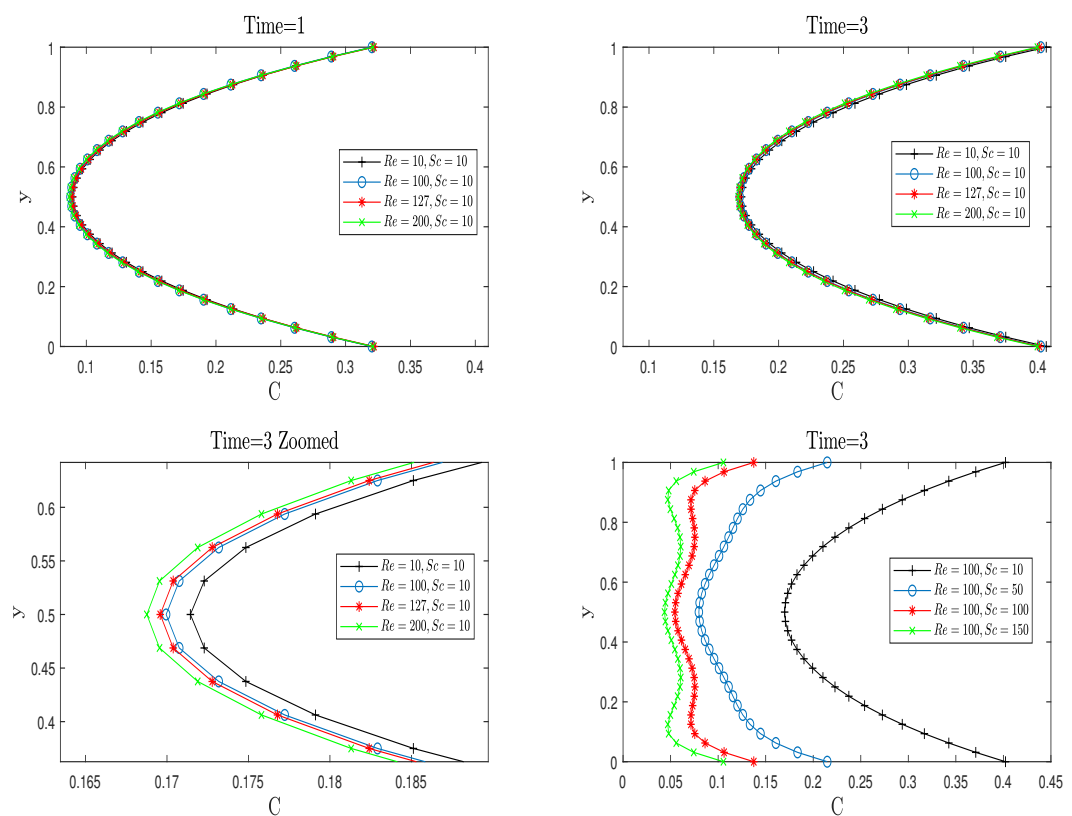

Fig. 9. Concentration along the vertical line through the geometric center of the square cavity at different Reynolds numbers $R e=10,100,127,200$ and Schmidt numbers $S c=10,50,100,150$ at different time level $t=1,3$

$R e=100$, the average Sherwood number is a positive real number for $S c=10$, while it becomes negative for $S c=50$ to 150 .
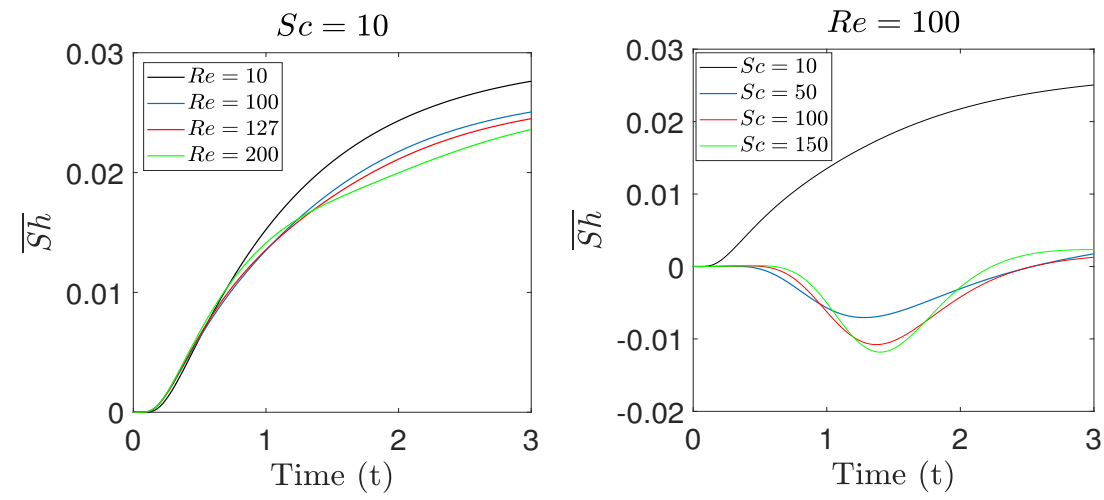

Fig. 10. Average Sherwood number for a four-sided lid-driven square cavity with different Reynolds numbers $R e=100-200$ and Schmidt numbers $S c=10-150$

The average Sherwood number increases and attains a steady-state solution for $S c=10$ at $t=3$, while it first decreases and then increases to attain a steady-state solution for $S c=50$ to 150 . The average Sherwood number becomes approximately zero after achieving a steady-state solution for $S c=50$ to 150 . 


\section{Code validation}

We have validated our present computer code for the high-Re solution for incompressible flow using a multi-grid method by Ghia et al. [2] (Figs. 11 and 12). Also, to determine the accuracy of our present numerical scheme, we have studied the four-sided, lid-driven square cavity problem investigated by Perumal and Dass [9], and Azwadi et al. [10].

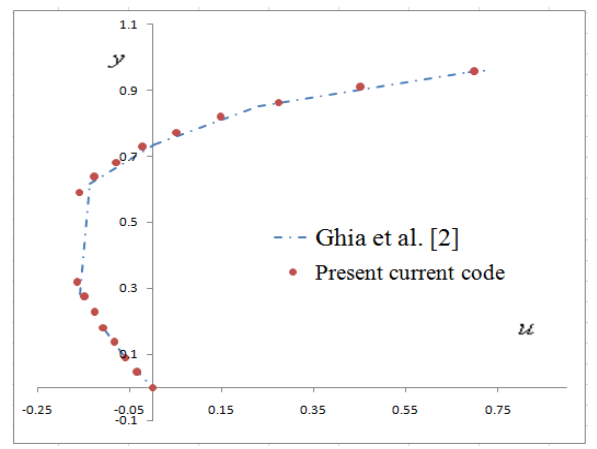

Fig. 11. $u$-velocity profiles along the vertical line through center-line of the square cavity at $R e=100$

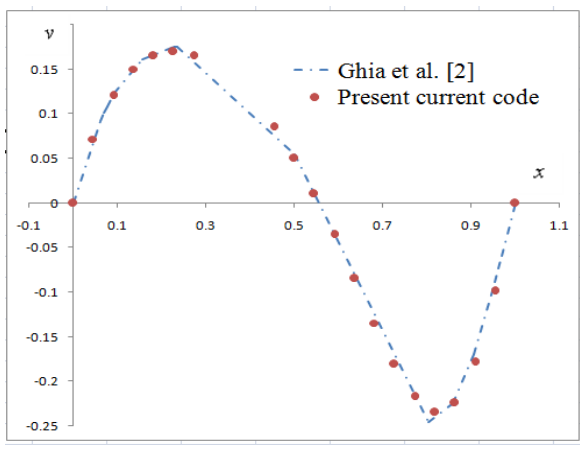

Fig. 12. $v$-velocity profiles along the horizontal line through center-line of the square cavity at $R e=100$

We have compared the locations of vortices which occur in the vicinity of four walls in Table 2. It is found that our results and those of $[9,10]$, are in good agreement.

Table 2. Comparison of centers of vortices at TPV, BPV, LPV and RPV of Perumal and Dass [9], Azwadi et al. [10] with present work at different Reynolds numbers

\begin{tabular}{|c|c|c|c|c|c|}
\hline $\begin{array}{c}\text { Reynolds } \\
\text { number }\end{array}$ & Reference & TPV & BPV & LPV & RPV \\
\hline \multirow{3}{*}{$\operatorname{Re}=10$} & Perumal and Dass [9] & $0.510,0.850$ & $0.490,0.149$ & $0.150,0.490$ & $0.849,0.510$ \\
& Azwadi et al. $[10]$ & $0.509,0.851$ & $0.497,0.155$ & $0.155,0.497$ & $0.851,0.509$ \\
& Present work & $0.509,0.849$ & $0.497,0.157$ & $0.155,0.497$ & $0.849,0.509$ \\
\hline \multirow{3}{*}{$\operatorname{Re}=100$} & Perumal and Dass [9] & $0.550,0.840$ & $0.450,0.160$ & $0.160,0.450$ & $0.840,0.550$ \\
& Azwadi et al. $[10]$ & $0.559,0.845$ & $0.442,0.161$ & $0.161,0.442$ & $0.845,0.559$ \\
& Present work & $0.560,0.842$ & $0.446,0.160$ & $0.160,0.446$ & $0.842,0.560$ \\
\hline \multirow{3}{*}{$\operatorname{Re}=127$} & Perumal and Dass [9] & $0.548,0.830$ & $0.449,0.168$ & $0.170,0.450$ & $0.830,0.550$ \\
& Azwadi et al. $[10]$ & $0.559,0.839$ & $0.442,0.168$ & $0.168,0.442$ & $0.839,0.559$ \\
& Present work & $0.567,0.840$ & $0.440,0.167$ & $0.167,0.440$ & $0.840,0.567$ \\
\hline
\end{tabular}

\section{Conclusions}

With the aid of numerical computations, we illustrate the variation of the $u$-velocity, temperature $(T)$, concentration $(C)$, average Nusselt number $(\overline{N u})$, and the average Sherwood number $(\overline{S h})$ along the vertical line and $v$-velocity along 
the horizontal line through the geometric center of the square cavity respectively. The effect of the Reynolds number and the Schmidt number have been demonstrated in the numerical computations. We can draw the following conclusions based on our numerical simulation:

- The four streamline vortices are generated namely, Left Primary Vortex (LPV), Right Primary Vortex (RPV), Bottom Primary Vortex (BPV) and Top Primary Vortex (TPV). The top and bottom primary vortices are moving in a clockwise direction, while the left and right primary vortices are moving in a anticlockwise direction. The center of these streamline vortices start shifting towards anti-diagonal as the Reynolds number increases from $R e=10$ to 127 .

- The direction and magnitude of the velocity profiles changes as we move towards the center of the cavity due to fluid circulation. The absolute value of the velocity profiles increases in the vicinity of the moving wall of the square cavity as the Reynolds number increased from $R e=10$ to 200 .

- The absolute value of vorticity along the diagonal as well as anti-diagonal of the square cavity increases with an increase of Reynolds number $R e=10$ to 200.

- The isotherm contours are symmetric about the horizontal line through the geometric center of the square cavity and are wavy. The temperature profiles increase with an increase of Reynolds number from $R e=10$ to 100 , while it decreases for $R e=100$ to 200 .

- The concentration contours appear to be symmetric about the horizontal line through the geometric center of the square cavity at $S c=10$, while the contours are the mirror image of each other about the horizontal and vertical line through the geometric center of the square cavity for Schmidt numbers $S c=50$ to 150 .

\section{Acknowledgment}

This work is supported by the University Grants Commission, Government ministry of India for providing a research grant for the second author with student id: MAY2018-421012. Furthermore, we would like to thank the referee for his/her valuable suggestions to improve the quality of the manuscript.

\section{References}

[1] Ambethkar, V., \& Kushawaha, D. (2017). Numerical simulations of fluid flow and heat transfer in a four-sided lid-driven rectangular domain. arXiv:1705.00707v1 [physics.fludyn] 26 Apr 2017.

[2] Ghia, U., Ghia, K.N., \& Shin, C.T. (1982). High-Re solutions for incompressible flow using the Navier-Stokes equations and a multigrid method. Journal of Computational Physics, 48 , 387-411, DOI: 10.1016/0021-9991(82)90058-4.

[3] Taylor, G.I. (1962). On Scraping Viscous Fluid from a Plane Surface. In: M. Shafer (ed.), Miszellangen der Angewandten Mechanik. Berlin: Akademie - Verlag, 313-315. 
[4] Ottino, J.M. (1989). The Kinematics of Mixing: Stretching, Chaos, and Transport. New York: Cambridge University Press.

[5] Luo, W.J., \& Yang, R.J. (2007). Multiple fluid flow and heat transfer solutions in a twosided lid-driven cavity. International Journal of Heat and Mass Transfer, 50, 2394-2405, DOI:10.1016/j.ijheatmasstransfer.2006.10.025.

[6] Ben-Nakhi, A., \& Chamkha, A.J. (2007). Conjugate natural convection around a finned pipe in a square enclosure with internal heat generation. International Journal of Heat and Mass Transfer, 50, 2260-2271, DOI:10.1016/j.ijheatmasstransfer.2006.10.036.

[7] Wahba, E.M. (2009). Multiplicity of states for two-sided and four-sided lid driven cavity flows. Computers and Fluids, 38, 247-253. DOI: 10.1016/j.compfluid.2008.02.001.

[8] Kumar, D.S., Dass, A.K., \& Dewan, A. (2012). Multiple stable solutions for two-and four-sided lid driven cavity flows using FAS Multigrid method. Engineering e-Transaction, 7(2), 96-106.

[9] Perumal, D.A., \& Dass, A.K. (2011). Multiplicity of steady solutions in two-dimensional liddriven cavity flows by lattice boltzmann method. Computer and Mathematics with Applications, 61, 3711-3721, DOI: 10.1016/j.camwa.2010.03.053.

[10] Azwadi, C.S.N., Rajab, A., \& Sofianuddin, A. (2014). Four-sided lid-driven cavity flow using time splitting method of Adams-Bashforth scheme. International Journal of Automotive and Mechanical Engineering (IJAME), 9, 1501-1510, DOI: 10.15282/ijame.9.2014.2.0124.

[11] Sivasankaran, S., Ananthan, S.S., Bhuvaneswari, M., \& Abdul Hakeem, A.K. (2017). Doublediffusive mixed convection in a lid-driven cavity with nonuniform heating on sidewalls. Indian Academy of Sciences, 42(11), 1929-1941, DOI: 10.1007/s12046-017-0735-4.

[12] Ambethkar, V., \& Kumar, M. (2017). Numerical solutions of 2-D unsteady incompressible flow with heat transfer in a driven square cavity using streamfunction-vorticity formulation. International Journal of Heat and Technology, 35(3), 459-473, DOI: 10.18280/ijht.350303.

[13] Romanò, F., Albensoeder, S., \& Kuhlmann, H.C. (2017). Topology of three-dimensional steady cellular flow in a two-sided anti-parallel lid-driven cavity. Journal of Fluid Mechanics, 826, 302-334, DOI: 10.1017/jfm.2017.422.

[14] Wu, H., Romanò, F., \& Kuhlmann, H.C. (2017). Attractors for the motion of finite-size particles in a two-sided lid-driven cavity. Proceedings in Applied Mathematics and Mechanics, 17(1), 669-670, DOI: 10.1002/pamm.201710303.

[15] Romano, F., Kunchi, K.P., \& Kuhlmann, H.C. (2019). Finite-size Lagrangian coherent structures in a two-sided lid-driven cavity. Phys. Rev. Fluids, 4(2), 024302, DOI: 10.1103/PhysRevFluids.4.024302.

[16] Kuhlmann, H.C., \& Romanò, F. (2019). The Lid-Driven Cavity. In: Gelfgat A. (eds), Computational Modelling of Bifurcations and Instabilities in Fluid Dynamics, Computational Methods in Applied Sciences, vol. 50, Cham: Springer.

[17] Romanò, F., Haotian, W., \& Kuhlmann, H.C. (2019). A generic mechanism for finite-size coherent particle structures. International Journal of Multiphase Flow, 111, 42-52.

[18] Ghoshdastidar, P.S. (1998). Computer Simulation of Flow and Heat Transfer. New Delhi: Tata McGraw-Hill Publishing Co. Ltd.

[19] Chapra, S.C., \& Canale, R.P. (1989). Numerical Methods for Engineers. New York: McGraw-Hill Companies.

[20] Smith, G.D. (1985). Numerical Solution of Partial Differential Equations: Finite Difference Methods. New York: Oxford University Press. 